\title{
Effect of Ilexonin A on the Notch signaling pathway following cerebral ischemia-reperfusion in rats.
}

\author{
Yu Han ${ }^{1,2}$, Guan-Yi Zheng1 ${ }^{*}$, Bi-Qin Zhang ${ }^{1}$, Xiao-Dong Chen ${ }^{1}$, Qiong Jiang1 \\ ${ }^{1}$ Fujian Medical University Union Hospital, Fuzhou, PR China \\ ${ }^{2}$ Huai' an First People Hospital, Huai'an, PR China
}

\begin{abstract}
Ilexonin A is a pentacyclic triterpenes, extracted from the herb Ilex pubescens with anti-thrombosis and anti-inflammatory activity, and improves blood circulation. This study determines whether Ilexonin A (IA) activates Notch signaling to induce neurogenesis following cerebral ischemia-reperfusion injury. We investigated the effect of Ilexonfound in the Ilexonin A-treatedin A on post-ischemic proliferation and differentiation of neural stem cells. Male Sprague-Dawley rats were divided into four groups: normal $(n=6)$, sham-operated $(n=6)$, control $(n=24)$, and IA-treated $(n=24)$. Focal cerebral ischemia-reperfusion injury was induced by the Middle Cerebral Artery Occlusion (MCAO) method. Ilexonin A at dose of 40 $\mathrm{mg} / \mathrm{kg}$ was administered via intraperitoneal injection immediately following MCAO and then once daily until the rats were sacrificed. 5-bromo 2-deoxyuridine labeled proliferating cells and neurogenesis and Notch induction in the ischemic tissue perienchyma were observed at $\mathrm{d} 1,3,7$, and 14 following ischemia by immunohistochemistry. NICD protein, Notch1, PS1, and HES1 gene expression was detected. Ilexonin A decreased the infarct volume and improved neurological deficits in a time-dependent manner. A higher number of Brdu/Nestin double-positive cells was found in the Ilexonin A-treated rats, compared with the rats exposed to ischemia only at both $3 \mathrm{~d}\left(14.33 \pm 1.63 \mathrm{vs} .11 .33 \pm 1.21,{ }^{*} \mathrm{p}<0.05\right)$ and $7 \mathrm{~d}\left(29.33 \pm 2.58\right.$ vs. $\left.24.50 \pm 2.7,{ }^{*} \mathrm{p}<0.05\right)$. Ilexonin A activates Notch signaling by increasing the expression of NICD at both $3 \mathrm{~d}\left(75.83 \pm 1.94\right.$ vs. $\left.69.67 \pm 3.93,{ }^{\#} \mathrm{p}<0.01\right)$ and $7 \mathrm{~d}(53.67 \pm 2.42 \mathrm{vs.} 50.17 \pm$ 2.93, ${ }^{\#} \mathbf{p}<0.01$ ). Ilexonin A also increased the gene expression levels of Notch1, PS1, and HES1 at $3 \mathrm{~d}$ after reperfusion in the rats. These findings suggested that the protective effects of Ilexonin $A$ were associated with neuronal regeneration, and the inactivation of Notch signaling pathways.
\end{abstract}

Keywords: Neurogenesis, Notch intracellular domain, Brdu, Nestin, Notch1, PS1, HES1.

Accepted on June 12, 2017

\section{Introduction}

Atopy is a condition where the immune system reacts to common substances in the environment (allergens) with a genetically determined overproduction of allergen specific antibodies of $\operatorname{IgE}$ isotype. This reaction is known as the type-I hypersensitivity reaction according to Coombs and Gell. The atopic phenotype can simply be characterized as the dominance of the Th-2 type over the Th1 type immune response. Allergic inflammation is the important part of the type-I allergic reaction. Why atopic individuals react to common allergens with the Th-2 mediated mechanism is not entirely known. Influence of colonisation with different intestinal microbiota during early infancy, impact of early infant nutrition, exposure to allergens and other little-known factors of prenatal and postnatal period are hypothesized. Neurogenesis occurs in two regions of adult mammalian brain: the Subventricular Zone (SVZ) of the lateral ventricle and the Subgranular Zone (SGZ) of the Dentate Gyrus (DG) in the hippocampus. New neural cells migrate either from the SVZ to the olfactory bulb via the rostral migratory stream and differentiate into neurons and glia or from the SGZ to the granule cell layer of the dentate gyrus [1]. Cerebral ischemia induces neuronal proliferation and neurogenesis to help in the recovery process from the ischemic injury [2-4]. However, the reason for proliferation of endogenous neuronal progenitors remains unknown. Currently, several possible mechanisms for post-ischemic neuronal proliferation have been proposed. Previous studies suggest that the classical Notch signaling pathway was responsible for proliferation and differentiation [5,6]. Neuronal cell death after brain ischemia triggers Notch signaling [7]. After stroke, increased levels of Notch and HES1 were associated with a significant increase in proliferating cells. Blockage of the Notch pathway significantly reduced the expression of Notch1, $N I C D$, and HES1 and subsequently the stroke-induced cell proliferation. Notch signaling was activated in the SVZ after focal ischemia and ischemia-induced cell proliferation in the SVZ could be blocked by inhibiting the Notch pathway in young adult brain [8]. Activation of Notch pathway results in infarct reduction and motor deficit improvement in aged 
ischemic rats [9]. Based on these findings, several studies have focused on the role of the Notch pathway in neurogenesis.

Notch is a fundamental pathway, which controls fate acquistion and plays a critical role in proliferation and differentiation of NSCs during brain development [5]. As a cell-surface receptor, Notch binds to its ligands, Jagged or Delta-like, and is processed in a two-step proteolytic cleavage. Following the second cleavage, mediated by an enzymatic complex formed by presenilin- 1 and $\gamma$-secretase, the Notch Intracellular Domain (NICD) is released and translocates to the nucleus, where it regulates the transcription of Notch targets, such as genes in the Hes family. This process is known as the canonical Notch signaling [10].

Ilexonin A is a compound extracted from the herb Ilex pubescens and can inhibit platelet aggregation and adhesion and reduce inflammation [11]. Studies have shown that during the early stage of diffuse encephalic swelling after stroke, high doses of Ilexonin A can increase microcirculation and improve the overall outcome [12]. Ilexonin A, which can reduce the infarct volume and activate the neurotrophic factor secretion, has neuroprotective potential after cerebral ischemia/ reperfusion injuries [13]. In the present study, we investigated the effect of Ilexonin A on the Notch signaling pathway by controlling NSC proliferation and differentiation, following cerebral ischemia/reperfusion. Thus, we explored a possible repair mechanism after brain injury. Our previous studies showed that Ilexonin A had best protective effect in the periinfarct region at a dose of $40 \mathrm{mg} / \mathrm{kg}$, and thus, we chose to carry out the experiments in this study by using that predetermined dose.

\section{Materials and Methods}

\section{Animal model}

Adult male Sprague-Dawley rats weighing $250 \pm 10 \mathrm{~g}$ were provided by the Experimental Animal Center of Fujian Medical University. They were maintained on a $12 \mathrm{~h}$ light/dark cycle with free access to food and water. Focal cerebral ischemia-reperfusion injury was induced by the Middle Cerebral Artery Occlusion (MCAO) method, based on the method developed by Longa et al. [14]. Briefly, rats were anesthetized by intraperitoneal injection of $10 \%$ chloral hydrate $(300 \mathrm{mg} / \mathrm{kg})$. A monofilament nylon suture (Diameter: $0.26 \mathrm{~mm}$ ) with rounded tip was introduced into the left internal carotid artery, into the external carotid artery, and advanced 18 $\pm 0.5 \mathrm{~mm}$ past the carotid bifurcation. After $2 \mathrm{~h}$ of ischemia, the nylon suture was withdrawn to allow reperfusion. The suture remained in place until the rats were sacrificed. Rats in the shame group received the same surgical procedures except the artery was not incised and occluded. Rats in the Ilexonin A group were treated with intraperitoneal injections of IA (40 $\mathrm{mg} / \mathrm{kg}$ ) immediately after MCAO and then once daily until the rats were sacrificed. Rats in the control group received intraperitoneally injections of saline. To label the population of proliferating cells, 5-bromo-2-deoxyuridine (BrdU, $50 \mathrm{mg} / \mathrm{kg}$,
Sigma-Aldrich, USA) was injected intraperitoneally at the onset of ischemia and then daily until the rats were sacrificed.

The severity of the neurological deficit was assessed at 1, 3, 7 and $14 \mathrm{~d}$ post-surgery according to the Zea-Longa neurological deficit score. A score 0 was given to normal animals with no signs of neurological deficit. A score 1 was given to those with mild neurological disorders, including dysfunction in stretching the left anterior limb. A score 2 was given to animals that intorted the left anterior limb and adducted the shoulder when they were lifted by the tail and also turned left when they walked. A score 3 was given to animals with neurologic abnormalities so severe that they fell to the left side when they walked. Rats with a score 4 did not walk spontaneously and had a depressed level of consciousness [15].

\section{Immunohistochemistry}

On the post-ischemia d 1, 3, 7, and 14, rats were perfused transcardially with normal saline, followed by $4 \%$ paraformaldehyde in PBS. Tissue was harvested, fixed overnight in $4 \%$ paraformaldehyde, and dehydrated in successive gradient sucrose concentrations $(15 \%, 20 \%$ and $30 \%$ ), until the specimens sink to the bottom. $8 \mu \mathrm{m}$-thick coronal sections were cut with a cryomicrotome (MICROM HM525, Germany). Sections were incubated in $2 \mathrm{~mol} / \mathrm{L} \mathrm{HCl}$ for $15 \mathrm{~min}$ at $37^{\circ} \mathrm{C}$, rinsed in $0.1 \mathrm{~mol} / \mathrm{L}$ boric acid $(\mathrm{pH} 8.4$ ) for $12 \mathrm{~min}$ at room temperature, followed by a PBS-T wash $(0.1 \%$ Triton X-100) for 15 min. Blocking solution goat serum (Zhongshan Biotechnology, Beijing, China) was carried out for $1 \mathrm{~h}$ at $37^{\circ} \mathrm{C}$. For double immunofluorescence, sections were incubated overnight at $4^{\circ} \mathrm{C}$ with primary antibodies: mouse monoclonal anti-BrdU antibody (1:200, Sigma-Aldrich, USA), rabbit polyclonal anti-NeuN (1:400, Chemicon, USA) or rabbit polyclonal anti-Nestin (1:50, Santa Cruz, USA). After a brief wash in PBS, they were incubated with Fluorescein Isothiocyanate (FITC) and Tetramethyl Rhodamine Isothiocyanate (TRITC) conjugated IgG for $2 \mathrm{~h}$ at room temperature. Sections were imaged by a fluorescent microscope (DMI3000B, Lecia, Germany). Sections incubated in PBS, instead of primary antibodies, served as negative controls.

\section{Western blotting}

The protein level of NICD in the ischemia zone was determined in the brains removed from six rats at each time point (d 1, 3, 7, and 14 post-ischemia). The ischemic tissue was mixed with cold protein extraction buffer, centrifuged at $10,000 \mathrm{rpm}$ for $10 \mathrm{~min}$ at $4^{\circ} \mathrm{C}$, and the supernatant was collected. Protein concentrations were measured by the BCA assay. Equal amounts of protein $(80 \mu \mathrm{g})$ were electrophoresed on a $10 \%$ SDS-PAGE gel and transferred to PVDF membrane for $2 \mathrm{~h}$ at $350 \mathrm{v}$. Following incubation with 5\% non-fat milk for $1 \mathrm{~h}$ at room temperature, membranes were incubated overnight at $4{ }^{\circ} \mathrm{C}$ with rabbit monoclonal anti-cleaved Notch1 (Val1747) antibody (1:1000). Membranes were then incubated with antirabbit horseradish peroxidase-conjugated $\operatorname{IgG}(1: 5000)$ for $2 \mathrm{~h}$ at room temperature. Immunoreactive bands quantitative 
analysis was performed by Micro Computer Imaging Device (MCID) software (Imaging Research, Leica, Germany) after scanning of the X-ray film with imaging software (Alpha Lnnotech Corporation, USA). The housekeeping protein actin was used as loading control.

\section{Reverse transcriptase-PCR (RT-PCR) assay}

Total RNA was isolated from post-ischemic brain tissue using TRlzol. RT-PCR was performed using TOYOBO First Strand cDNA synthesis Kit and ReverTraAce according to the manufacturer's instructions. cDNA was synthesized using oligo-dT primers. PCR was performed with specific primers. The product $(5 \mu \mathrm{L})$ was analyzed on $2 \%$ agarose electrophoretic gels, stained with Gen Green and visualized by ImageMaster $^{\circledR}$ VDS. The density of each band was quantified by using the Image 1.59 software. All results represent the average density of positive bands, obtained from six independent experiments using different reaction cycles. The PCR primers and $\beta$-actin were developed by Ding Guo biological technology. The sequences of the primers were as follows:

\section{Notch1 forward: 5-CTGGTTCCCTGAGGGTTTCAA-3}

reverse: 5-GGAACTTCTTGGTCTCCAGGT-3

\section{PS1 forward: 5-TAATGGCCCGACCCCAGAGTA-3}

reverse: 5-CAATCATCCCGACCACACCA -3

\section{Hes 1 forward: 5- AAAGACAGCCTCTGAGCACA-3}

reverse: 5-TCATGGCGTTGATCTGGGTCA-3

\section{$\beta$-actin forward: 5-CACCCGCGAGTACAACCTTC-3}

reverse: 5-CCCATACCCACCATCACACC-3

Cycling conditions were as follows: For Notch1 the initial melting temperature was $94^{\circ} \mathrm{C}$ for $2 \mathrm{~min}$, followed by 35 cycles at $94^{\circ} \mathrm{C}$ for $30 \mathrm{~s}, 55^{\circ} \mathrm{C}$ for $30 \mathrm{~s}, 72^{\circ} \mathrm{C}$ for $30 \mathrm{~s}$, with a final extension at $72^{\circ} \mathrm{C}$ for $10 \mathrm{~min}$. For PS1 the initial melting temperature was $94^{\circ} \mathrm{C}$ for $2 \mathrm{~min}$, followed by 35 cycles at $94^{\circ} \mathrm{C}$ for $30 \mathrm{~s}, 58^{\circ} \mathrm{C}$ for $30 \mathrm{~s}, 72^{\circ} \mathrm{C}$ for $30 \mathrm{~s}$, with a final extension at $72^{\circ} \mathrm{C}$ for $10 \mathrm{~min}$. For Hesl the initial melting temperature was $94^{\circ} \mathrm{C}$ for $2 \mathrm{~min}$, followed by 35 cycles at $94^{\circ} \mathrm{C}$ for $30 \mathrm{~s}, 58^{\circ} \mathrm{C}$ for $30 \mathrm{~s}, 72^{\circ} \mathrm{C}$ for $30 \mathrm{~s}$, with a final extension at $72^{\circ} \mathrm{C}$ for $10 \mathrm{~min}$. For $\beta$-actin the initial melting temperature was $94^{\circ} \mathrm{C}$ for $2 \mathrm{~min}$, followed by 35 cycles at $94^{\circ} \mathrm{C}$ for $30 \mathrm{~s}, 56^{\circ} \mathrm{C}$ for $30 \mathrm{~s}, 72^{\circ} \mathrm{C}$ for $30 \mathrm{~s}$, with a final extension at $72^{\circ} \mathrm{C}$ for $10 \mathrm{~min}$. For quantification purposes, the expression of all genes was normalized against $\beta$-actin and results were expressed as relative fold change of Notch $1 / \beta$ actin, PS1/ $\beta$-actin, and Hes $1 / \beta$-actin.

\section{Statistical analysis}

All data are expressed as the mean \pm SD and analysed by oneway ANOVA using the SPSS 17.0 software package. Differences between groups were analysed using a LSD test for homogeneity of variance and a Games-Howell test for heterogeneity of variance. $\mathrm{P}<0.05$ was considered to be statistically significant.

\section{Results}

\section{IA injections reduces brain injury and decreases the neurological deficit after cerebral ischemia- reperfusion}

When assessing the neurological severity scores rats in the normal and sham groups had similar behavior before and after surgery, while rats in the model and IA groups developed unilateral weakness after surgery, a neurological deficit that gradually diminished over time. Compared with the control group, the IA group had significantly better Neurological Severity Scores (NSS) at all-time points $(\mathrm{p}<0.01, \mathrm{p}<0.05)$ (Figure 1A). Triphenyl Tetrazolium Chloride (TTC) staining revealed a pale area in the striatum and cortex of the postischemic brains, representing the infarct region. The infarct volume (white region) decreased significantly following $3 \mathrm{~d}$ treatment with IA (Figure 1B).

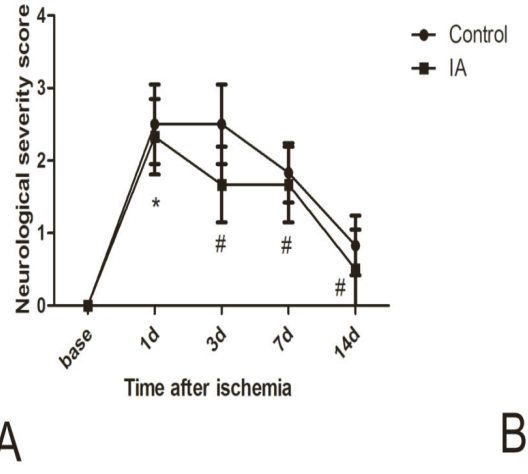

Control 3d IA 3d

Figure 1. Neurological severity score and infarct. A. Effect of IA on the neurological function, as evaluated by the neurological severity score. Values are mean $\pm S D ; n=6$ per time point $\left(^{*} p<0.05\right)$. B. TTC staining of post-ischemia rat brains.

\section{Post-ischemia IA treatment promotes cortical neurogenesis}

Nestin is a marker for Neural Stem Cells (NSC) proliferation, used to determine the neurogenesis status. Unlike the normal group, the control and IA groups have increased numbers of BrdU/Nestin double-positive cells, which peaked at $7 \mathrm{~d}$ after cerebral ischemia-reperfusion. In addition, the number of positive cells in the IA group was significantly higher than those in the control group at both $3 \mathrm{~d}(14.33 \pm 1.63$ vs. $11.33 \pm$ $\left.1.21,{ }^{*} \mathrm{p}<0.05\right)$ and $7 \mathrm{~d}\left(29.33 \pm 2.58\right.$ vs. $\left.24.50 \pm 2.7,{ }^{*} \mathrm{p}<0.05\right)$ (Figure 2). This suggests that no NSC proliferation in brain cortex before ischemia, IA enhanced endogenous NSCs proliferation in the cortex after ischemia. 


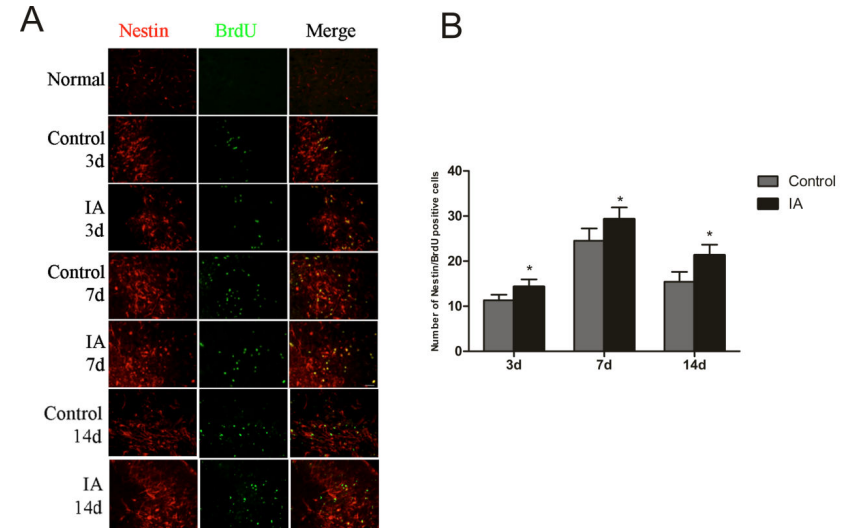

Figure 2. A. Double immunofluorescence staining for BrdU and Nestin in ipsilateral brain tissue after MCAO. Images of stained tissue sections from control or IA-treated rats. B. Number of BrdU/ Nestin double-positive cells at each time point. Values are mean \pm $S D ; n=6$ per time point ( ${ }^{*} p<0.05 \mathrm{vs}$. control).

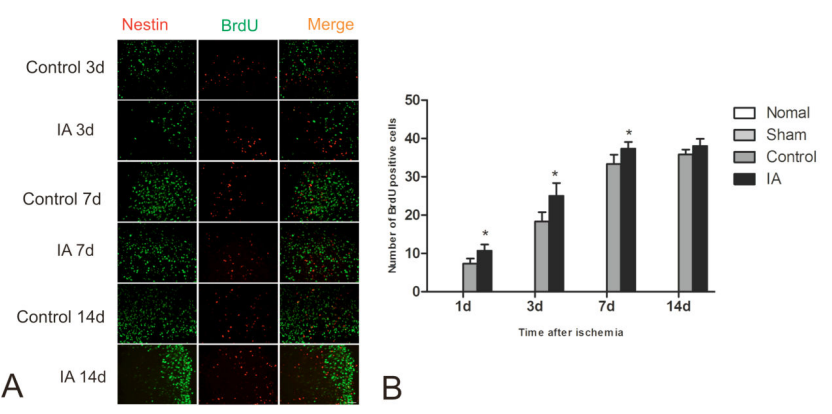

Figure 3. A. Double immunofluorescence staining for BrdU and NeuN in ipsilateral brain tissue after MCAO. Control or IA-treated brains at $3 d, 7 d$, and $14 d$ after ischemia. Green: NeuN immunoreactivity; Red: BrdU immunoreactivity; Yellow: BrdU/NeuN double immunofluorescence. Bar $=20 \mu \mathrm{m}$. B. Quantitative data show number of BrdU positive cells in the four experimental groups. Values are mean $\pm S D ; n=6$ per time point $\left(^{*} p<0.05\right.$ vs. control).

\section{Proliferating cells differentiate from NSC}

BrdU-positive cells were observed in ischemic cortices at $\mathrm{d} 1$ after cerebral ischemia-reperfusion in both control and IA groups. The number of positive cells increased gradually and still continued to rise around d 14 . There were significant statistical differences between the control and IA groups $\left({ }^{*} \mathrm{p}<0.05\right)$. Additionally, a few double-positive BrdU/NeuN cells were detected at $\mathrm{d} 14$, suggesting that the BrdU positive cells differentiated from NSC (Figure 3).

\section{Ischemia increases NICD levels after reperfusion}

To further evaluate the activation of Notch signaling, we determined the levels of NICD, a Notch activation marker, in the four experimental groups. As show in the immunofluorescent detection of NICD, positive cells focused in the perienchyma ischemia. NICD protein express in both cytoplasm and nucleus (Figure 4A). In control and IA groups, the number of NICD positive cells was elevated one day after reperfusion, reached a maximum at $\mathrm{d} 3$ and started decreasing around $\mathrm{d} 7$, to return to pre-reperfusion levels (Figure 4B). The number of NICD positive cells in the ischemic cortex increased remarkably after $3 \mathrm{~d}\left(75.83 \pm 1.94\right.$ vs. $69.67 \pm 3.93,{ }^{*} \mathrm{p}<0.01$, (Figure $4 \mathrm{C})$ ) and $7 \mathrm{~d}\left(53.67 \pm 2.42\right.$ vs. $50.17 \pm 2.93,{ }^{*} \mathrm{p}<0.01$, (Figure 4C)). Western bloting analysis of the NICD protein in the ischemic penumbra after MCAO showed that NICD protein increased at $\mathrm{d} 1\left({ }^{*} \mathrm{p}<0.05\right)$, peaked at $\mathrm{d} 3$ and remained high at $14 \mathrm{~d}\left({ }^{*} \mathrm{p}<0.05\right)$ after ischemia (Figure 4D).
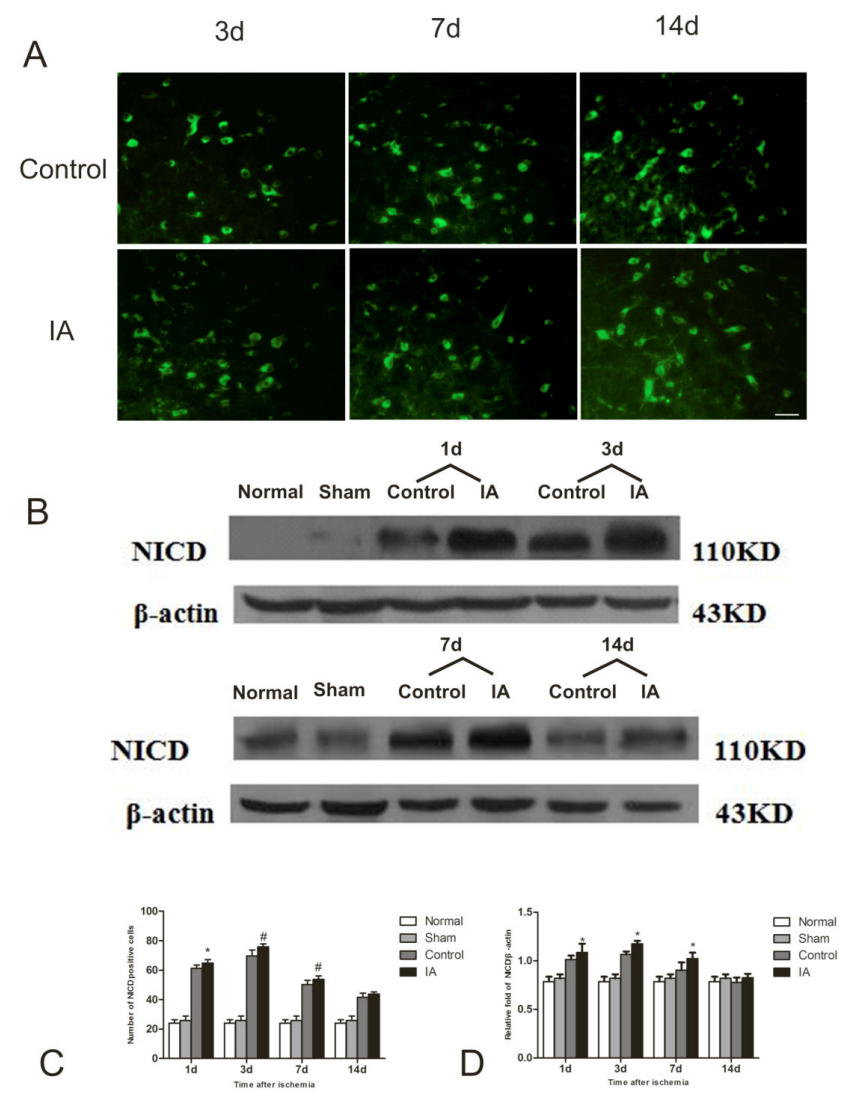

Figure 4. A. NICD immunofluorescence in the ipsilateral brain tissue after MCAO. Cells collected from control or IA-treated rat brains. $B$. NICD protein (110 kDa) expressions (measured by western blotting) in four groups at four time points. C. Number of NICD positive cells in the four experimental groups. D. Relative fold change in $N I C D / \beta$ actin in the four groups.

\section{IA activates Notch signaling}

We investigated the effect of IA on Notch signaling by measuring the expression levels of Notch1, PS1, and HES1 (Figure 5). The levels increased gradually in the control and IA groups, peaking at $3 \mathrm{~d}$ after reperfusion. Additionally, there was a statistical significance between control and IA groups at all $\left({ }^{*} \mathrm{p}<0.05\right.$ versus control ${ }^{\#} \mathrm{p}<0.01$ versus control).

\section{Discussion}

Neurogenesis is a process that occurs throughout mammalian is responsible for maintenance of various brain functions [16]. Cerebral ischemia is known to stimulate neurogenesis $[17,18]$. Since NSC or progenitor cells are dormant in the adult brain and can be activated by injury. NSC proliferation in the SVZ was highly activated by ischemia and newly generated neurons were functionally integrated into the neuronal circuitry [19]. It 
has been reported that Ilexonin A has therapeutic effects in ischemia by improving microcirculation and preventing inflammation and thrombosis. Moreover, it has neuroprotective potential by enhancing the secretion of neurotrophic factors and attenuating cerebral edema [13]. Results have shown that the infarction area was focused to the striatum and dorsolateral region of the cerebral cortex. The NSS decreased after treatment with Ilexonin A, suggesting that IA improves the recovery of neurological functions. Nestin-positive cells begin to accumulate in the peri-ischemic regions at $\mathrm{d} 1$ after the stroke in the control and IA groups, in thread-like structures emanating from blood vessels or "budding" from capillary ends [20]. Unlike the control and IA groups, in the normal and sham groups, there were only few Nestin-positive cells, which marked microvascularization. Compared with the control group, in the IA group we observed an increased number of $\mathrm{Brdu} / \mathrm{Nestin}$ double-positive cells, which appeared at the ischemic border at around $3 \mathrm{~d}$ post-stroke and were maintained for a week, to gradually starting to decrease thereafter. This confirms the findings in a previous study, which shows that the number of Brdu/Nestin cells increased remarkably in the SVZ, hippocampus, striatum, and neocortex during the first week after MCAO [2]. Our results suggest that cerebral ischemia induces NSC proliferation and the IA treatment is maintaining it.

$\mathrm{Brdu} / \mathrm{NeuN}$ double-positive cells were detected only around $\mathrm{d}$ 14 and no significant difference was observed between the control and IA groups. This could have several explanations. First, the BrdU dose or uptake time was insufficient to show NSC differentiation. Second, new NSC may differentiate into glial cells instead of neurons. Fujiki study revealed that adult NSC differentiate into mature neurons only in SVZ and SGZ, while in the area surrounding the ischemia zone, they generally differentiate into glial cells [21]. Our research has suggested that astrocytes and oligodendrocytes were increased in the perienchyma of ischemic tissue after cerebral ischemiareperfusion, and that they were differentiated from Brdu/Nestin positive cells. Glial scar can inhibit axonal regeneration and astrocytes have been considered a barrier to neural repair. However, emerging studies suggest that glia suppress the invasion of immune cells into the peri-infarct region [22], suggesting that they are not only protecting neurons in the acute phase of cerebral ischemia, but also promoting neurogensis and angiogenesis in the chronic phase [23,24]. Reactive astrocytes in peri-ischemic cortex may promote neurovascular remodeling, which becomes useful for the functional recovery after stroke [25]. IA plays an important role in the structural repair of brain tissue after cerebral ischemia. At the same time, further research on the role of glial cells in the differentiation of neural stem cells is necessary.

Notch signaling plays a crucial role in maintaining the balance between Neural Stem Cells (NSCs) and Neural Progenitor Cells (NPCs), which supply the brain with specific neural populations, both under normal conditions and after injury [26]. Transient activation of Notch is responsible for maintenance and differentiation of neural progenitors, shortly after brain injury [27]. The activated Notch signaling compensates for neuronal loss and promotes postmitotic neuronal growth at the onset of neurodegeneration [28]. In the present research, we found the number of NICD positive cells to increase at day one and reach a maximum at $3 \mathrm{~d}$ after ischemia, which suggests that Notch signaling was activated by cerebral ischemia-reperfusion. Expression of Notch1, PS1, and HES1 genes and NICD protein is upregulated in of the area surrounding the ischemic tissue, an additional evidence for ischemia-activated Notch signaling. It was shown that Notch inhibition by siRNA results in decreased expression of NICD and HES1 and reduced NSC proliferation after stroke [8]. Correlation analysis showed that the numbers of Brdu/Nestin and NICD positive cells show significant correlation at $3 \mathrm{~d}$ $(\mathrm{r}=0.916, \mathrm{p}<0.05)$ and $7 \mathrm{~d}(\mathrm{r}=0.916, \mathrm{p}<0.01)$ post-ischemia. This suggests that Notch signaling pathway mediates neural stem cells proliferation after cerebral ischemia-reperfusion.

A

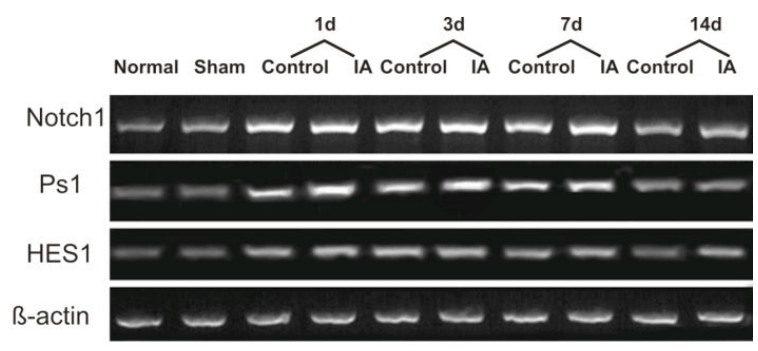

B

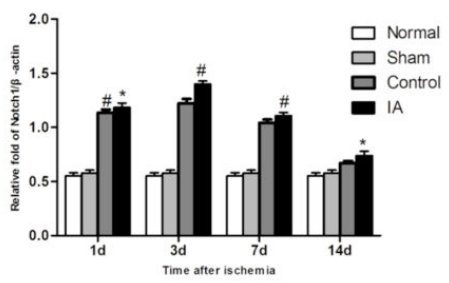

C
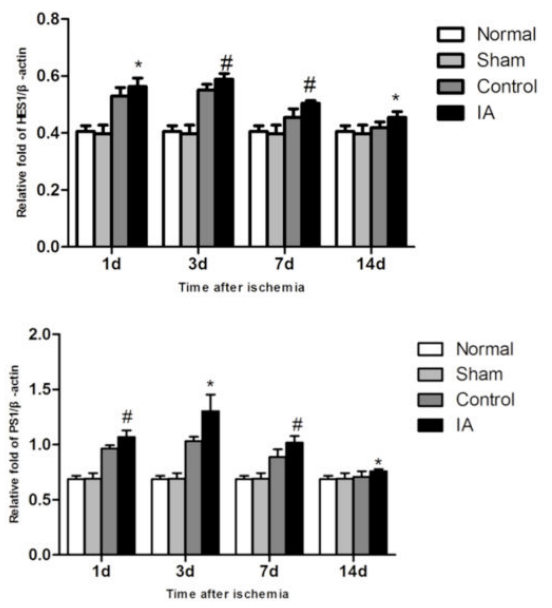

Figure 5. Notch1, PS1, and HES1 gene expression in all four groups at four time-points. A. Image of Notch1, PS1, and Hesl gene expression. B. Relative fold change in Notch1/ $\beta$-actin. C. Relative

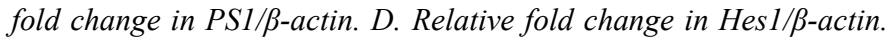
Values are mean $\pm S D ; n=6$ per time point $\left(^{*} p<0.05\right.$ vs. control; ${ }_{p} p<0.01$ vs. control).

In recent years, several study explored ways to influence Notch signal in order to provide new therapeutics. Currently, research in the field suggested that folic acid and soybean isoflavone 
can potentially induce Notch signaling to promote neurogenesis and reduce neuronal apoptosis [29,30]. Here we show that intraperitoneal injections of IA, immediately after ischemia-reperfusion and continuing for $14 \mathrm{~d}$, result in increased levels of NICD protein and Notch1, PS1, and HES1 gene expression, suggesting that IA promotes Notch activation.

Ischemia causes damage to the brain tissue and induces neural regeneration and angiogenesis, a process in which Notch also plays a role. It is known to be a required factor for the physiological remodeling of the retinal vasculature [31]. Simvastatin increases arteriogenesis after stroke and may work by upregulating PS1 and increasing the activity of Notch [32]. High levels of miR-210 can stimulate Notch activation and thus contribute to angiogenesis after cerebral ischemia [33]. After stroke, angiogenesis is necessary to not only restrain the ischemic zone, but also to supply a nutrient-rich environment for NSC proliferation and migration. SVZ-derived NPC migrate along blood vessels from the SVZ toward the ischemic region of the striatum, an area closely associated with blood vessels, which function as scaffold for NPCs migration toward the damaged brain region [34]. Following cerebral injury, endothelial cells of the cerebral vessels secret SDF-1 $\alpha$ to attract NSC expressing CXCR4, which promotes migration of NSC to the ischemic border [35]. It has been confirmed that IA stabilizes the vascular endothelium, thus improving microcirculation and inducing capillary hyperplasia to relieve cerebral edema.

Therefore, IA and Notch signaling have a synergistic action on the NSCs proliferation. But it is necessary to investigate whether the effect of IA on Notch signaling is responsible for increased angiogenesis, which additionally promotes neurogenesis.

\section{Acknowledgments}

This work was supported by Program for New Century Excellent Talents in Fujian Province University, China (No NCETFJ-0704), Professorial Academic Development Foundation of Fujian Medical University (JS09014), and Subject of Medical Innovation in Fujian Province (2012CX-16) and Natural Science Foundation of Fujian, China (grant no. 2014J01327). The authors thank Clarity Manuscript Consultant LLC (Indianapolis) for their language editing.

\section{References}

1. Hoehn BD, Palmer TD, Steinberg GK. Neurogenesis in rats after focal cerebral ischemia is enhanced by indomethacin. Stroke 2005; 36: 2718-2724.

2. Shin HY, Kim JH, Phi JH, Park CK, Kim JE. Endogenous neurogenesis and neovascularization in the neocortex of the rat after focal cerebral ischemia. J Neurosci Res 2008; 86: 356-367.

3. Fishell G, Goldman JE. A silver lining to stroke: does ischemia generate new cortical interneurons? Nat Neurosci 2010; 13: 145-146.
4. Kahle MP, Bix GJ. Neuronal restoration following ischemic stroke: influences, barriers, and therapeutic potential. Neurorehabil Neural Repair 2013; 27: 469-478.

5. Artavanis-Tsakonas S, Rand MD, Lake RJ. Notch signaling: cell fate control and signal integration in development. Science 1999; 284: 770-776.

6. Borlongan $\mathrm{CV}$, Lind JG, Dillon-Carter O, Yu G, Hadman $\mathrm{M}$, Cheng C, Carroll J, Hess DC. Bone marrow grafts restore cerebral blood flow and blood brain barrier in stroke rats. Brain Res 2004; 1010: 108-116.

7. Tatsumi K, Okuda H, Makinodan M, Yamauchi T, Makinodan E. Transient activation of Notch signaling in the injured adult brain. J Chem Neuroanat 2010; 39: 15-19.

8. Wang L, Chopp M, Zhang RL, Zhang L, Letourneau Y, Feng YF, Jiang A, Morris DC, Zhang ZG. The Notch pathway mediates expansion of a progenitor pool and neuronal differentiation in adult neural progenitor cells after stroke. Neuroscience 2009; 158: 1356-1363.

9. Sun F, Mao X, Xie L, Ding M, Shao B, Jin K. Notch1 signaling modulates neuronal progenitor activity in the subventricular zone in response to aging and focal ischemia. Aging cell 2013; 12: 978-987.

10. Zhao Y, Chen X, Ma L, Zuo Z, Zhu Z, Zhu X, Wang Q, He E, Xiong L, Pei J, Xu L, Hou L, Chen S. Electroacupuncture pretreatment induces tolerance against focal cerebral ischemia through activation of canonical Notch pathway. BMC Neurosci 2012; 13: 111.

11. Hua HY, Li YY. Ilexonin A studies on pharmacological effect. Chin J Modern Med 2006; 8: 137-138.

12. Sheng HL, Dong XL, Jiang YF. Protective effects of Radix Ilicis Pubescentis extract on focal cerebra 1 ischemia reperfusion injury in rats. Chin J New Drugs 2009; 18: 1020-1022.

13. Zheng GY, Shi WQ. Influence of Ilexonin A on the expression of bFGF, GAP-43 and neurogenesis after cerebral ischemia-reperfusion in rats. Acta Pharmaceutica Sinica 2011; 46: 1065-1071.

14. Longa EZ, Weinstein PR, Carlson S, Cummins R. Reversible middle cerebral artery occlusion without craniectomy in rats. Stroke 1989; 20: 84-91.

15. Tang Q, Han R, Xiao H, Shi L, Shen J, Lun Q, Li J. Role of suture diameter and vessel insertion position in the establishment of the middle cerebral artery occlusion rat model. Exp Ther Med 2013; 5: 1603-1608.

16. Imayoshi I, Kageyama R. The role of Notch signaling in adult neurogenesis. Mol Neurobiol 2011; 44: 7-12.

17. Marti-Fabregas J, Romaguera-Ros M, Gomez-Pinedo U, Martinez-Ramirez S, Jimenez-Xarrie RME, R JLM-V, Garcia-Verdugo JM. Proliferation in the human ipsilateral subventricular zone after ischemic stroke. Neurology 2010; 74: $357-365$.

18. Delavaran H, Sjunnesson H, Arvidsson A, Lindvall O, Norrving B, van Westen D, Kokaia Z, Lindgren A. Proximity of brain infarcts to regions of endogenous neurogenesis and involvement of striatum in ischaemic stroke. Eur J Neurol 2013; 20: 473-479. 
19. Ohira K, Furuta T, Hioki H, Nakamura KC, Kuramoto E, Tanaka Y, Funatsu N, Shimizu K, Oishi T, Hayashi M, Miyakawa T, Kaneko T, Nakamura S. Ischemia-induced neurogenesis of neocortical layer 1 progenitor cells. Nat Neurosci 2010; 13: 173-179.

20. Popa-Wagner A, Dinca I, Yalikun S, Walker L, Kroemer H. Accelerated delimitation of the infarct zone by capillaryderived nestin-positive cells in aged rats. Curr Neurovasc Res 2006; 3: 3-13.

21. Fujiki M, Abe E, Nagai Y, Shiqi K, Kubo T, Ishii K, Abe T, Kobayashi H. Electroconvulsive seizure-induced VEGF is correlated with neuroprotective effects against cerebral infarction: Involvement of the phosphatidylinositol-3 kinase/Akt pathway. Exp Neurol 2010; 225: 377-383.

22. Shimada IS, Borders A, Aronshtam A, Spees JL. Proliferating reactive astrocytes are regulated by Notch-1 in the peri-infarct area after stroke. Stroke 2011; 42: 3231-3237.

23. Shen LH, Li Y, Chopp M. Astrocytic endogenous glial cell derived neurotrophic factor production is enhanced by bone marrow stromal cell transplantation in the ischemic boundary zone after stroke in adult rats. Glia 2010; 58: 1074-1081.

24. Xin $\mathrm{H}$, Li Y, Shen LH, Liu X, Wang X, Zhang J, Pourabdollah-Nejad DS, Zhang C, Zhang L, Jiang H, Zhang ZG, Chopp M. Increasing tPA activity in astrocytes induced by multipotent mesenchymal stromal cells facilitate neurite outgrowth after stroke in the mouse. PLoS ONE 2010; 5: 9027.

25. Hayakawa K, Nakano T, Irie K, Higuchi S, Fujioka M, Orito K, Iwasaki K, Jin G, Lo EH, Mishima K, Fujiwara M. Inhibition of reactive astrocytes with fluorocitrate retards neurovascular remodeling and recovery after focal cerebral ischemia in mice. J Cereb Blood Flow Metab 2010; 30: 871-882.

26. Aguirre A, Rubio ME, Gallo V. Notch and EGFR pathway interaction regulates neural stem cell number and selfrenewal. Nature 2010; 467: 323-327.

27. Androutsellis-Theotokis A, Leker RR, Soldner F, Hoeppner DJ, Ravin R, Poser SW, Rueger MA, Bae SK, Kittappa R,
McKay RD. Notch signalling regulates stem cell numbers in vitro and in vivo. Nature 2006; 442: 823-826.

28. Yuan TM, Yu HM. Notch signaling: key role in intrauterine infection/inflammation, embryonic development, and white matter damage? J Neurosci Res 2010; 88: 461-468.

29. Huang G, Cao X, Zhang X, Chang H, Yang Y, Du W, Wilson JX. Effects of soybean isoflavone on the notch signal pathway of the brain in rats with cerebral ischemia. $\mathrm{J}$ Nutr Sci Vitaminol 2009; 55: 326-331.

30. Zhang X, Huang G, Liu H, Chang H, Wilson JX. Folic acid enhances Notch signaling, hippocampal neurogenesis, and cognitive function in a rat model of cerebral ischemia. Nutr Neurosci 2012; 15: 55-61.

31. Ehling M, Adams S, Benedito R, Adams RH. Notch controls retinal blood vessel maturation and quiescence. Development 2013; 140: 3051-3061.

32. Zacharek A, Chen J, Cui X, Yang Y, Chopp M. Simvastatin increases notch signaling activity and promotes arteriogenesis after stroke. Stroke 2009; 40: 254-260.

33. Lou YL, Guo F, Liu F, Gao FL, Zhang PQ. miR-210 activates notch signaling pathway in angiogenesis induced by cerebral ischemia. Mol Cell Biochem 2012; 370: 45-51.

34. Kojima T, Hirota Y, Ema M, Takahashi S, Miyoshi I, Okano H, Sawamoto K. Subventricular zone-derived neural progenitor cells migrate along a blood vessel scaffold toward the post-stroke striatum. Stem Cells 2010; 28: 545-554.

35.Xiong Y, Mahmood A, Chopp M. Angiogenesis, neurogenesis and brain recovery of function following injury. Curr Opin Investig Drugs 2010; 11: 298-308.

\section{*Correspondence to}

Guan-Yi Zheng

Fujian Medical University Union Hospital

Fuzhou

PR China 